\title{
Estudio Intercultural de una Batería Sociocognitiva de Autoeficacia Vocacional
}

\author{
Intercultural Study of a Socio-Cognitive Battery of Vocational Self-Efficacy
}

\author{
Gina Chávez-Ventura ${ }^{1}$, Henry Santa-Cruz-Espinoza ${ }^{2}$, César Merino-Soto ${ }^{3}$, Maricela Osorio- \\ Guzmán $^{4}$, Julio Jaime-Salas ${ }^{5}$ y Alicia E. Risueño ${ }^{6}$
}

\begin{abstract}
Resumen
En Latinoamérica existe la necesidad de disponer de instrumentos basados en el modelo teórico sociocognitivo de desarrollo de carrera, por su respaldo científico y utilidad práctica. Por ello, el objetivo del estudio fue construir una batería sociocognitiva de autoeficacia vocacional para estudiantes de Psicología y obtener sus evidencias de validez de contenido. Participaron 21 jueces psicólogos de Argentina, Colombia, México y Perú, quienes valoraron los criterios de coherencia y relevancia. También, 40 estudiantes de Psicología de los últimos semestres académicos, de los países mencionados, valoraron la claridad de los ítems. Luego de una evaluación cuantitativa mediante la V de Aiken, se obtuvo una batería de 95 ítems, cuyos límites inferiores en los intervalos de confianza fueron iguales o superiores a .62. Finalmente, se dispone de una batería que permitirá ampliar los estudios en Psicología vocacional y cuyas evidencias de validez basadas en la estructura interna deberán investigarse.
\end{abstract}

Palabras clave: teoría social cognitiva de carrera, validez de contenido

\begin{abstract}
In Latin America there is a need for instruments based on the socio-cognitive theoretical model of career development, due to its scientific support and practical usefulness. Therefore, the objective of the study was to build a socio-cognitive battery of vocational self-efficacy for psychology students and obtain their validity evidence of content. 21 psychologist judges from Argentina, Colombia, Mexico and Peru participated, who assessed the criteria of coherence and relevance. Also, 40 psychology students of the last academic semesters, from the countries mentioned, valued the clarity of the items. After a quantitative evaluation using Aiken V, a battery of 95 items was obtained, whose lower limits on confidence intervals were equal to or higher than .62. Finally, there is a battery that will allow to expand studies in vocational psychology and whose validity evidence based on the internal structure should be investigated.
\end{abstract}

Keywords: social cognitive career theory, content validity

\footnotetext{
${ }^{1}$ Doctora en Psicología. Docente Investigadora de la Universidad César Vallejo, Perú. http://orcid.org/0000-0002-4638-3487. Scopus Author ID: 56175307800. Universidad César Vallejo. Vicerrectorado de Investigación. Av. Larco 1770. Urb. Las Flores, distrito Víctor Larco, Trujillo, Perú. Tel.: +51999191641. Correo: gina.chavezv@ hotmail.com

${ }^{2}$ Magíster en Educación, Teorías y Práctica Educativa. Docente de la Universidad César Vallejo, Perú. http://orcid.org/0000-00026475-9724. Scopus Author ID: 57197585194. Universidad César Vallejo. Vicerrectorado de Investigación. Av. Larco 1770. Urb. Las Flores, distrito Víctor Larco, Trujillo, Perú. Tel: +51999192447. Correo: hsantacruze@ gmail.com

${ }^{3}$ Maestro en Psicología. Docente Investigador de la Universidad San Martín de Porres, Lima, Perú. http://orcid.org/0000-0002-14078306. Scopus Author ID: 34870294000. Calle Vieja 332, interior 116, Condominio Bugambilias 2, Cuernavaca, Morelos. Tel.: 527774259409. Correo: sikayax@yahoo.com.ar

${ }^{4}$ Doctora de Investigación en Ciencias Psicológicas y Pedagógicas. Profesora e Investigadora Titular C. de la Universidad Nacional Autónoma de México, FES Ixtacala. http://orcid.org/0000-0001-7798-5301. Scopus Author ID: 6504000176. Universidad Nacional Autónoma de México. Facultad de Estudios Superiores Iztacala. Jefatura de Psicología. Av. de los Barrios 1, los Reyes Iztacala. Tlalnepantla Estado de México. 54090 México. Tel.: (55) 56231333 ext.39806. Correo: mosorio@unam.mx

${ }^{5}$ Doctor en Ciencias Sociales, Niñez y Juventud. Docente de la Universidad Surcolombiana, Colombia. Avda. Pastrana con Cdra. 1 Universidad Surcolombiana, Neiva, Huila, Colombia. Tel.: +573155578860. Correo: julio.jaime@usco.edu.co

${ }^{6}$ Doctora en Psicología. Secretaria de Evaluación y Acreditación de la Universidad Kennedy, Argentina. Sarachaga 4949, Buenos Aires, Argentina. Tel.: +5491145388665. Correo: aliciamas@ fibertel.com.ar
} 


\section{Introducción}

El alto rendimiento de los universitarios es uno de los indicadores objetivos de la excelencia en su formación profesional; sin embargo, la calidad involucra también aspectos no tangibles, como el compromiso con la calidad académica (García-Jiménez, 2016). Así, en el ámbito del desempeño, la autoeficacia cumple un papel importante, debido a que impulsa el esfuerzo, la atención, la perseverancia en demandas específicas y en dominios de conducta particulares (Bandura, 1982), además, establece metas adaptativas y el esfuerzo para su consecución (Wolf, Herrmann, \& Brandstätter, 2018). Este concepto, con base en la teoría sociocognitiva de Bandura (1977, 1986, 1997), asume una perspectiva de la agencia personal, referida a la dirección y autorregulación del propio comportamiento mediante la previsión, manifestada en las creencias sobre lo que las personas son capaces de hacer, lo que alcanzarán como resultado de sus acciones, sus metas planteadas y la implementación para alcanzarlas; todo ello en interacción con los sistemas sociales (Bandura, 1991), que imponen limitaciones o recursos para el desenvolvimiento personal y moldean culturalmente las creencias y propósitos (Bandura, 2008).

La teoría sociocognitiva de Bandura se ha aplicado al ámbito educativo (Zimmerman, Bandura, \& Martínez-Pons, 1992), de salud (Bandura, 1998), del deporte (Beauchamp, Crawford, \& Jackson, 2018), de gestión organizacional (Wood, \& Bandura, 1989), así como al de desarrollo de carrera (Lent, Brown, \& Hackett, 1994; Lent, Ezeofor, Morrison, Penn, \& Ireland, 2016); sin embargo, diversos estudios, entre ellos instrumentales (Aydin \& Pasinlioglu, 2018; Domínguez-Lara, 2017a; Porter, Keefe, Wellington \& de Williams, 2008; TschannenMoran \& Hoy, 2001), han abordado en particular una de sus variables, la autoeficacia, por el rol preponderante que desempeña en la motivación humana (Bandura, 2010).

El enfoque social cognitivo de desarrollo de carrera busca explicar cómo las personas durante su vida se desarrollan y desenvuelven en la preparación, elección (Lent, Brown, \& Hackett, 1994; Lent, Hackett, \& Brown, 2004), desempeño profesional (Lent, Brown, \& Hackett, 1994), satisfacción (Lent \& Brown, 2006) y autogestión en la carrera (Lent \& Brown, 2013), desde distintos modelos explicativos, con supuestos empíricamente comprobables que otorgan sustento a la teoría, permiten su aplicabilidad y generalización (Lent, 2017). Plantea que las personas se formulan metas, como consecuencia de sus intereses permanentes en las actividades, asociadas a su percepción de competencia (autoeficacia) y de resultados positivos de sus expectativas (Lent et al., 1994).

El modelo asumido, denominado autoeficacia vocacional, se focaliza en el estudiante en formación profesional de Psicología que realiza prácticas de su carrera profesional, afirmando su vocación y vivenciando desempeños "previos" al ejercicio profesional, donde también puede experimentar "satisfacciones" por su elección. Así, en el presente estudio, se resaltarán algunos conceptos relevantes a la construcción de una batería del modelo social cognitivo: autoeficacia, expectativas de resultado, metas, intereses, apoyo y barreras.

La autoeficacia percibida es el juicio de las propias capacidades para organizar y ejecutar determinados dominios de desempeño (Lent, 2005; Lent, Brown, \& Hackett, 2002; Lent et al., 1994). Se adquiere mediante la interacción de los logros de desempeño, el aprendizaje experiencialobservacional y vicario, la persuasión verbal, y el estado fisiológico y afectivo (Bandura, 1986; Brusokas \& Malinauskas, 2014); esta interacción afecta a los juicios de rendimiento e influyen en la conducta (Lent \& Hackett, 1987). Además, un reporte de investigación muestra que la autoeficacia en la elección profesional es mediadora de la eficacia de la pasantía y preparación profesional en estudiantes universitarios (Chen-Tsang, Hsuan, \& Chun-Chin, 2017).

Otra variable que forma parte del modelo es: expectativas de resultado o consecuencias imaginadas de comportamientos específicos (Lent et al., 1994) y pueden ser de tipo: físico, social y autoevaluativo (Bandura, 1986). Éstas influyen en el inicio de un nuevo comportamiento y en su mantenimiento, pudiendo ser afectadas por las barreras que se presenten (Leung, 2008). 
Las metas son la determinación para participar de actividades o producir un resultado futuro (Bandura, 1986); posibilitan la autorregulación del individuo y organizan el comportamiento de acuerdo a ellas (Lent et al., 1994); también pueden ser impulsadas por el apoyo y obstaculizadas por las barreras, procedentes del contexto (Lent, Hackett, \& Brown, 2004). El apoyo proveniente de padres y maestros aumentan la autoeficacia para la toma de decisiones profesionales, que a su vez favorece el optimismo profesional (García, Restubog, Bordia, \& Roxas, 2015); mientras que las barreras internas percibidas son más importantes que las externas al valorar el ajuste vocacional; y, la adaptabilidad de la carrera, media la relación entre las barreras y los resultados alcanzados en universitarios (Urbanaviciute, Pociute, Kairys, \& Liniauskaite, 2016).

Los intereses promueven los objetivos de carrera y aumentan la probabilidad de elección de comportamientos, dominios de desempeño y logros; pueden servir de apoyo a la eficacia y a las expectativas de resultado (Lent et al., 1994). Son importante predictor de la transición a la formación profesional (Volodina \& Nagy, 2016).

Por otra parte, los estudios instrumentales o explicativos en los que se han construido instrumentos en relación al modelo sociocognitivo de desarrollo de carrera han dirigido su atención, especialmente al proceso de elección de carrera (Betz \& Taylor, 2012; Blanco, Casas, \& Mafokozi, 2016; Carbonero \& Merino Tejedor, 2002; Lent, Brown, \& Larkin, 1986; Lent et al., 2003; Makransky, Rogers, \& Creed, 2014; Miguel, Silva, \& Prieto, 2013); siendo en su mayoría de procedencia americana anglosajona y en idioma inglés, por lo que su aplicación en otros contextos está limitada (Casas \& Blanco-Blanco, 2016; Sheu \& Bordon, 2017), lo cual se ve reflejado en la escasa investigación llevada a cabo en Latinoamérica y en habla hispana.

Las limitaciones también consisten en la centralidad cultural de la elaboración de los constructos y de los indicadores para representarlos. Esto conduce a la sustantividad del constructo, esto es, su significancia conceptual, tenga predominante relevancia para la cultura en que se originó, pero no necesariamente para otros grupos culturalmente diferentes. En contraste con la elaboración culturalmente centrada, la práctica recomendada es que, el proceso de construcción de una medida de uso generalizado intercultural, consista en descentrar culturalmente el de construcción de la medida, mediante un proceso simultáneo de desarrollo de la definición del constructo y de los ítems (Tanzer, 2005). Por lo tanto, una medida que produzca puntajes con significancia en varias culturas, se ajusta a la justicia (fairness) de una medida (Kane, 2013).

Además, en la actualidad es relevante y necesario obtener evidencia de la aplicabilidad de la teoría y establecer el nexo entre teoríainvestigación-práctica en el campo de dominio profesional y de desarrollo de carrera (Ali \& Brown, 2017; Blustein, 2017; Howard, 2017; Lent, 2017), así como evaluar los cambios suscitados en el tiempo (Lent, 2017), para comprender, explicar y abordar los problemas prácticos de las experiencias de desarrollo vocacional y de trabajo; para lo cual, los psicólogos cumplen un rol importante que les exige adaptarse a las nuevas demandas sociales, a fin de promover cambios positivos de las personas con las que trabajan (Ali \& Brown, 2017).

Obtener evidencia de la eficacia de la labor realizada por los profesionales de la Psicología, implica dirigir la atención a las competencias logradas en su formación profesional (González, González, \& Vicencio, 2014; Lunt, Peiró, Poortinga, \& Roe, 2015; Red Psicología Proyecto MECESUP ULS 0601, 2013) que se hacen visibles en el desempeño de las prácticas preprofesionales. Por tanto, disponer de algún instrumento de medición de la autoeficacia vocacional que pueda predecir dicho desempeño en estudiantes de Psicología, que próximamente se insertarán en el mundo laboral, contribuiría a ofrecer una evidencia empírica al modelo social cognitivo de desarrollo de carrera que articule la teoría-investigación-práctica; y, además, pueda ser una herramienta que oriente la toma de decisiones en favor de la calidad de la formación profesional.

Por lo expuesto, este estudio inicial se orienta a mostrar las evidencias de validez de contenido de una batería diseñada para medir la autoeficacia vocacional, que abarque las variables del modelo social cognitivo de carrera, en estudiantes que están por concluir la carrera de Psicología, de los países de Argentina, Colombia, México y Perú. 
Además, será posible ofrecer una medida que sirva de apoyo para la generación de nuevos estudios desde la Psicología vocacional. Este aspecto del estudio de la validez es importante para corroborar el muestreo del contenido respecto al alcance de su extensión, la importancia comparada con su capacidad predictiva de criterios conductuales, y la claridad expresada en sus indicadores (ítems). Como una fase inicial en el estudio de la validez, el enfoque intercultural de su construcción permite respaldar la generalización de su medición. Para el presente estudio, se valoró la importancia de recurrir también al grupo-objetivo hacia el cual va dirigido el instrumento, específicamente para evaluar la claridad del contenido, debido a la experiencia directa con el contenido construido y la discrepancia observada entre ellos y jueces al valorar la claridad de los ítems (Merino-Soto, 2016).

\section{Método}

\section{Participantes}

Con un muestreo no probabilístico de tipo intencional, se eligieron dos grupos de participantes, jueces expertos y estudiantes universitarios. Respecto al primer grupo, en la fase preliminar del estudio, se contó con la participación de 8 jueces psicólogos, de los cuales 7 eran peruanos y 1 mexicana, con las siguientes características: 1 varón especialista en orientación y desarrollo de carrera, radicado en Estados Unidos; 7 (6 varones y 1 mujer) con más de 10 años de ejercicio en la carrera y docentes universitarios, con experiencia en investigación, en el campo clínico, educativo, organizacional o social.

Luego de la fase preliminar, se solicitó la participación de 21 jueces, con los siguientes criterios: ser psicólogos (52\% mujeres y $48 \%$ varones), con desempeño en docencia universitaria formando profesionales de la Psicología, con conocimientos auto-informados en la temática, experiencia en investigación y/o psicometría (docencia o publicaciones relevantes), y disponibilidad. En cada país participante, los jueces seleccionados pertenecían a las instituciones de los autores; la muestra se distribuyó del siguiente modo: 5 Argentina, Colombia y Perú (5 en cada uno), y 6 en México.

El segundo grupo consistió de 40 estudiantes de Psicología, elegidos con los siguientes criterios: estar en los últimos semestres académicos (entre 5 y 6 años de estudio), y que estuvieran realizando prácticas pre-profesionales. Estos criterios permitirían identificar participantes con más experiencia acumulada y oportunidades para contrastar lo aprendido con su aplicación en situaciones pre-laborales. Éstos valoraron únicamente la claridad de los ítems. El promedio de edad es de 25.6 años (mín.=20; máx.=50) y el $80 \%$ son mujeres.

\section{Instrumento}

Batería Socio-cognitiva de Autoeficacia vocacional. Incluye 6 escalas (autoeficacia percibida, expectativas de resultado, intereses, metas, barreras y apoyo) diseñadas en el formato de una prueba de autoinforme, debido que se consideró un medio válido de medición (Domínguez-Lara, 2017b), especialmente en una población que es capaz de expresar sus propias creencias y experiencias vinculadas a la carrera profesional. Respecto a los términos teóricos básicos, primero se definieron los constructos de acuerdo a la teoría asumida; y se construyó una Tabla de especificaciones, con dimensiones (escalas), indicadores e ítems.

De este modo, la autoeficacia vocacional se definió como la confianza en la propia capacidad para desempeñarse exitosamente en su carrera profesional que, sumada a las expectativas del resultado del ejercicio profesional, da lugar a intereses y metas que favorecen el desempeño.

La totalidad de los ítems se redactaron en sentido positivo, evitando doble negaciones y el efecto del método (Abad et al., 2004; Muñiz \& Fonseca-Pedrero, 2008). Se eligieron cinco opciones de respuesta debido que esa cantidad, en pruebas de ejecución típica, muestra suficiente varianza en la identificación de constructos y reduce la influencia de fuentes de error (Abad et al., 2004).

El contenido de las alternativas de respuesta de los ítems fueron respecto a la intensidad de la confianza (de total desconfianza hasta total desconfianza), del acuerdo (de completamente de acuerdo hasta completamente en desacuerdo), de 
afirmación (definitivamente sí hasta definitivamente no), interés (total desinterés hasta total interés), de apoyo (total apoyo hasta ningún apoyo), e intensidad general (totalmente hasta ninguno). De ese modo, el contenido de las opciones y de los constructos estuvo asociado, para facilitar la orientación de la respuesta.

\section{Procedimiento}

El estudio fue instrumental (Ato, López, \& Benavente, 2013) y se ha centrado en la obtención de la validez del contenido, procurando una equivalencia conceptual y lingüística interculturalmente, por considerarse esencial en el proceso de construcción de instrumentos (Carretero-Dios \& Pérez, 2007).

\section{Evaluación de jueces}

En la fase preliminar del estudio, 8 jueces revisaron de manera independiente y vía correo electrónico, la versión inicial de la prueba conformada por 87 ítems, en un formato en el que podían valorar de manera dicotómica ( $\mathrm{Si}-\mathrm{No}$ ) la claridad y la pertinencia de las dimensiones, indicadores e ítems, con la teoría, así como proporcionar alguna observación, si lo consideraban conveniente. Luego, en una siguiente revisión, constataron el levantamiento de las observaciones en 11 ítems corregidos y evaluaron 16 ítems agregados.

También los coautores revisaron la batería en su totalidad; es decir, en sus definiciones, información teórica, estructura y valoraron cuantitativamente los ítems de acuerdo a los criterios de claridad, coherencia y relevancia, mientras que la suficiencia se valoró cualitativamente, ya que cuando consideraron pertinente, señalaron los contenidos que faltaban abarcar en las dimensiones. Producto de la versión preliminar del instrumento se obtuvo un total de 95 ítems.

Se remitió la batería, de manera independiente a 21 jueces psicólogos de Argentina, Colombia, México y Perú en un formato vía correo electrónico, que fue devuelto por el mismo medio y que contenía las instrucciones: «Luego de presentarle la información sobre la prueba, encontrará una serie de ítems para evaluarlos según la claridad, coherencia y relevancia; posteriormente se le solicitará que agregue -si es necesario- algún indicador o ítem por cada dimensión. A continuación se presentan los criterios, que deberán valorarse con una puntuación de 1 a 7 ».

La claridad implica evaluar si el ítem es comprensible; la coherencia consistió en determinar si el ítem representa adecuadamente la dimensión que lo integra; y, la relevancia se refiere a si el ítem es importante o pertinente para evaluar el dominio elegido. Tales criterios se aplicaron para lograr la precisión y objetividad en la valoración de expertos (Muñiz \& FonsecaPedrero, 2008).

El formato para la evaluación del instrumento, contenía: la denominación de la prueba, su objetivo, a quiénes está dirigida, una síntesis de la fundamentación teórica con la definición de la variable, sus dimensiones y las referencias. Luego, se indicaron nuevas instrucciones para completar el formato: «A continuación valore cada ítem, según la claridad (si es comprensible), coherencia (si se relaciona con la dimensión/ indicador) y relevancia (si es importante), eligiendo el número que indique su apreciación. Recuerde que el número 1 indica nula claridad/coherencia/ relevancia y el número 7 refleja total claridad/coherencia/ relevancia». Vale señalar que por cada ítem, el jurado tenía la posibilidad de realizar comentarios o sugerencias, debajo de la columna "Observaciones". Al finalizar la presentación de cada dimensión, las instrucciones indicaban la posibilidad de incluir otro indicador o ítem considerado relevante.

Luego de haber incorporado las sugerencias del equipo internacional de jueces, 20 ítems fueron aceptados, 29 se agregaron, 51 se corrigieron y 24 se eliminaron. Por ello, en la segunda fase de revisión de los jueces, se obtuvo un instrumento con 100 ítems.

\section{Evaluación de estudiantes}

Se procedió a solicitar a 10 estudiantes de cada país participante, que valoraran individualmente la claridad de los ítems. La evaluación se llevó a cabo en aulas de clase, en condiciones adecuadas de iluminación, ventilación; y, en grupos de 10 estudiantes. Luego de conocer el objetivo de la evaluación y firmar el consentimiento informado, la participación fue anónima y voluntaria. 
Tabla 1. Estructura de la Batería Socio-cognitiva de Autoeficacia Vocacional

\begin{tabular}{|c|c|c|}
\hline Escalas & Indicadores & Ítems \\
\hline $\begin{array}{l}\text { Autoeficacia percibida. Confianza en que las } \\
\text { habilidades, características y conocimientos }\end{array}$ & $\begin{array}{l}\text { Confianza en las habilidades socioemocionales para la } \\
\text { interacción social. }\end{array}$ & $1-6$ \\
\hline \multirow{4}{*}{ adquiridos ayudarán en su praxis profesional. } & Confianza en la habilidad para diagnosticar. & $7-11$ \\
\hline & Confianza en la habilidad comunicativa & $17-23$ \\
\hline & Confianza en la capacidad de investigación & $24-32$ \\
\hline & Confianza en la ética profesional & $42-46$ \\
\hline \multirow{3}{*}{$\begin{array}{l}\text { Expectativas de resultado. Creencias personales } \\
\text { sobre los resultados imaginados que obtendrá en } \\
\text { el ejercicio profesional }\end{array}$} & Físico o tangible & $47-52$ \\
\hline & Social & $53-55$ \\
\hline & Auto-evaluativo o personal & $56-58$ \\
\hline $\begin{array}{l}\text { Metas. Determinación o intentos para } \\
\text { involucrarse en acciones que permitan conseguir } \\
\text { un resultado esperado en un futuro. }\end{array}$ & Determinación a la acción & $59-62$ \\
\hline $\begin{array}{l}\text { Intereses. Atracción y agrado por la carrera } \\
\text { profesional elegida y satisfacción por las } \\
\text { actividades que su ejercicio implica. }\end{array}$ & Agrado por el ejercicio profesional & $71-74$ \\
\hline \multirow{3}{*}{$\begin{array}{l}\text { Apoyo. Soporte obtenido para la consecución de } \\
\text { metas }\end{array}$} & Familiar & $75-77$ \\
\hline & Académico & $78-81$ \\
\hline & Social & $82-84$ \\
\hline \multirow{3}{*}{$\begin{array}{l}\text { Barreras. Obstáculos para la consecución de } \\
\text { metas }\end{array}$} & Familiar & $85-88$ \\
\hline & Académico & $89-92$ \\
\hline & Social & $93-95$ \\
\hline
\end{tabular}

El formato de evaluación de la prueba contenía las siguientes instrucciones: "Estamos en el proceso de construcción de una prueba psicológica y has sido seleccionado para que nos comuniques si los ítems que se te presentan a continuación son comprensibles. Al lado de cada ítem, elige por favor una puntuación entre 1 y 7 , donde el 1 indica que el ítem no es comprensible y el 7 es totalmente comprensible. Recuerda que lo que tienes que hacer es marcar el número, según te parezca claro o no, de cada ítem y si tienes alguna observación puedes hacerla. Tu apoyo será sumamente valioso. ¡Muchas gracias!”. También se incluyó una columna para que los estudiantes anotaran -si era el caso- alguna observación. Luego de que los estudiantes entregaron los formatos, se revisó si los cuestionarios estuvieron respondidos por completo y se agradeció la participación.

\section{Análisis de datos}

El análisis de la validez de contenido consistió en aplicar para cada ítem el coeficiente V (Aiken, 1980, 1985; Escurra, 1988) y para cada escala, se obtuvo el promedio del mismo (Moscoso \& Merino, 2017). Se halló la significancia estadística de V; sin embargo, la decisión de mantener o no los ítems se hizo mediante los intervalos de confianza (Penfield \& Giacobbi, 2004), porque permiten considerar valores mínimamente aceptables para las evidencias de validez de contenido (Merino \& Livia, 2009). Para el estudio se consideró .62 como valor mínimo del límite inferior del intervalo de confianza, apoyado en los siguientes argumentos: a) dicho valor supera el cálculo aleatorio; b) maximiza la cantidad de ítems retenidos, a fin de que el análisis factorial se realice sobre una mayor cantidad de ítems que adicionen mayor variabilidad en el contenido; y, c) los niveles mínimos basados en la literatura sugieren mucha discrepancia (Charter, 2003; Cicchetti, 1994; Domínguez-Lara, 2017b; Merino \& Livia, 2009; Merino-Soto, 2016).

\section{Resultados}

Para la decisión de la versión final de la batería, se consideró que el nivel de comprensión de los ítems no es equiparable entre la percepción de jueces expertos y estudiantes (Merino-Soto, 2016); además, la población blanco está conformada por estudiantes de Psicología de los últimos semestres académicos; por tanto, ellos deben valorar la inteligibilidad de los ítems (Domínguez-Lara, 2017a; Merino-Soto, 2016).

Con base a los límites inferiores de los intervalos de confianza $(\geq .62)$, se eliminaron cinco ítems (E1, E2, E3, E4 y E5); de los cuales, los tres primeros pertenecían a la escala de Autoeficacia percibida, el penúltimo a expectativas de resultado 
Tabla 2. Resumen de la evaluación de la claridad de los ítems, desde la percepción de los estudiantes

\begin{tabular}{|c|c|c|c|c|c|c|c|c|c|}
\hline \multirow{2}{*}{ Escalas } & & \multicolumn{2}{|c|}{ Argentina } & \multicolumn{2}{|c|}{ Colombia } & \multicolumn{2}{|c|}{ México } & \multicolumn{2}{|c|}{ Perú } \\
\hline & & $\mathrm{V}_{\text {Aiken }}$ & IC $95 \%$ & $\mathrm{~V}_{\text {Aiken }}$ & IC 95\% & $\mathrm{V}_{\text {Aiken }}$ & IC $95 \%$ & $\mathrm{~V}_{\text {Aiken }}$ & IC $95 \%$ \\
\hline \multirow{3}{*}{$\begin{array}{l}\text { Autoeficacia } \\
\text { percibida }\end{array}$} & Mín. & .82 & $.67-.91$ & .80 & $.65-.90$ & .80 & $.65-.90$ & .85 & $.71-.93$ \\
\hline & Máx. & 1.00 & $.91-1.00$ & 1.00 & $.91-1.00$ & .98 & $.88-1.00$ & 1.00 & $.91-1.00$ \\
\hline & Prom & .99 & $.89-.99$ & .95 & $.83-.98$ & .93 & $.81-.97$ & .97 & $.87-.99$ \\
\hline \multirow{3}{*}{$\begin{array}{l}\text { Expectativa } \\
\text { de resultado }\end{array}$} & Mín. & 1.00 & $.91-1.00$ & .92 & $.79-.97$ & .88 & $.73-.95$ & .97 & $.86-.99$ \\
\hline & Máx. & 1.00 & $.91-1.00$ & .92 & $.79-.97$ & .97 & $.86-.99$ & 1.00 & $.91-1.00$ \\
\hline & Prom & 1.00 & $.91-1.00$ & .92 & $.79-.97$ & .94 & $.82-.98$ & .99 & $.89-1.00$ \\
\hline \multirow[t]{3}{*}{ Metas } & Mín. & 1.00 & $.90-1.00$ & .97 & $.86-.99$ & .90 & $.77-.96$ & .97 & $.86-.99$ \\
\hline & Máx. & 1.00 & $.90-1.00$ & 1.00 & $.91-1.00$ & .93 & $.81-.98$ & 1.00 & $.91-1.00$ \\
\hline & Prom & 1.00 & $.90-1.00$ & .99 & $.89-1.00$ & .92 & $.79-.97$ & 1.00 & $.91-1.00$ \\
\hline \multirow[t]{3}{*}{ Intereses } & Mín. & 1.00 & $.91-1.00$ & 1.00 & $.91-1.00$ & .90 & $.77-.96$ & .90 & $.77-.96$ \\
\hline & Máx. & 1.00 & $.91-1.00$ & 1.00 & $.91-1.00$ & .97 & $.86-.99$ & 1.00 & $.91-1.00$ \\
\hline & Prom & 1.00 & $.91-1.00$ & 1.00 & $.91-1.00$ & .94 & $.83-.98$ & .98 & $.88-.99$ \\
\hline \multirow[t]{3}{*}{ Ароуо } & Mín. & 1.00 & $.91-1.00$ & .88 & $.75-.95$ & .82 & $.67-.91$ & .97 & $.86-.99$ \\
\hline & Máx. & 1.00 & $.91-1.00$ & .97 & $.86-.99$ & .93 & $.81-.98$ & 1.00 & $.91-1.00$ \\
\hline & Prom & 1.00 & $.91-1.00$ & .95 & $.84-.99$ & .89 & $.76-.95$ & 1.00 & $.90-1.00$ \\
\hline \multirow[t]{3}{*}{ Barreras } & Mín. & 1.00 & $.91-1.00$ & .95 & $.83-.99$ & .88 & $.75-.95$ & .90 & $.77-.96$ \\
\hline & Máx. & 1.00 & $.91-1.00$ & 1.00 & $.91-1.00$ & .93 & $.81-.98$ & 1.00 & $.91-1.00$ \\
\hline & Prom & 1.00 & $.91-1.00$ & .99 & $.89-1.00$ & .91 & $.79-.97$ & .98 & $.89-.99$ \\
\hline
\end{tabular}

y el último a intereses. Los ítems E1 (qué tanta confianza tengo para identificar los criterios para poder diagnosticar con certeza) y E3 (qué tanta confianza tengo para realizar planes para la mejora de la salud mental) no alcanzaron valores aceptables en el criterio de claridad percibida por los estudiantes mexicanos.

El ítem E2 tampoco cumplía el criterio de claridad en los estudiantes argentinos porque contenía el término "consejería psicológica", vocablo que para los estudiantes argentinos tiene una acepción negativa debido a que esta actividad la realizan personas que no son psicólogos y están fuera de la ley del ejercicio profesional, dicho término se reemplazó por "brindar orientación psicológica". Debido a este cambio el ítem se sometió a nueva valoración de jueces psicólogos y estudiantes.

También, debido a los comentarios de estudiantes argentinos, se adaptó el ítem 16 de la escala de Autoeficacia percibida, a los términos que se utilizan en su país, adicionando al ítem "qué tanta confianza tengo en mi capacidad para aplicar psicoterapia”, una barra (forward slash) y luego la expresión: "intervenir psicológicamente".

El ítem E4 (pienso que con el ejercicio de mi profesión conseguiré financiarme el perfeccionamiento profesional futuro), de la escala Expectativas de resultado, tampoco alcanzó el valor mínimo esperado en claridad para los estudiantes mexicanos. Similarmente, el ítem E5 (qué tanto me interesa escribir sobre mis experiencias en la profesión), de la escala Intereses, no obtuvo el límite inferior requerido en el criterio de relevancia de los jueces mexicanos. Eliminados los ítems inválidos, la estructura de la batería de 95 ítems, se presenta en la Tabla 1.

Considerando la amplia extensión de la batería y los criterios sometidos a evaluación en los 4 países participantes del estudio, aquí sólo se mostrarán los hallazgos de los promedios de $\mathrm{V}$ de Aiken y los intervalos de confianza de cada escala del instrumento.

Los hallazgos obtenidos en el criterio de claridad muestran que la $\mathrm{V}$ de Aiken de los ítems se encuentra entre .80 [.65-.90] y 1.00 [.91-1], con promedios más elevados en Argentina y menores en México; además, los valores de la $\mathrm{V}$ de Aiken y sus intervalos de confianza por escalas, difiere entre cada país. Las escalas con mayor valoración en claridad son Metas e Intereses (Tabla 2).

Considerando el criterio de coherencia percibida por los jueces se encontró que el promedio de las valoraciones de la $\mathrm{V}$ de Aiken quienes pertenecen a Argentina y Perú es 1.00 [.84-1], mientras que el promedio de las calificaciones de los jueces de México (.97 [.811]) es levemente menor y el promedio de Colombia (.83 [.62-.94]) es el más bajo. Además, el promedio por escalas es muy similar, con leve 
Tabla 3. Resumen de la evaluación de la coherencia de los ítems, desde la percepción de los jueces

\begin{tabular}{|c|c|c|c|c|c|c|c|c|c|}
\hline \multirow{2}{*}{ Escalas } & & \multicolumn{2}{|c|}{ Argentina } & \multicolumn{2}{|c|}{ Colombia } & \multicolumn{2}{|c|}{ México } & \multicolumn{2}{|c|}{ Perú } \\
\hline & & $\mathrm{V}_{\text {Aiken }}$ & IC $95 \%$ & $\mathrm{~V}_{\text {Aiken }}$ & IC $95 \%$ & $\mathrm{~V}_{\text {Aiken }}$ & IC $95 \%$ & $\mathrm{~V}_{\text {Aiken }}$ & IC $95 \%$ \\
\hline \multirow{3}{*}{$\begin{array}{l}\text { Autoeficacia } \\
\text { percibida }\end{array}$} & Mín. & 1.00 & $.84-1.00$ & .83 & $.62-.94$ & .83 & $.64-.93$ & 1.00 & $.81-1.00$ \\
\hline & Máx. & 1.00 & $.84-1.00$ & 1.00 & $.84-1.00$ & 1.00 & $.86-1.00$ & 1.00 & $.84-1.00$ \\
\hline & Prom & 1.00 & $.84-1.00$ & .99 & $.83-1.00$ & .97 & $.81-.99$ & 1.00 & $.84-1.00$ \\
\hline \multirow{3}{*}{$\begin{array}{l}\text { Expectativa } \\
\text { de resultado }\end{array}$} & Mín. & 1.00 & $.84-1.00$ & .97 & $.79-1.00$ & .97 & $.81-.99$ & 1.00 & $.84-1.00$ \\
\hline & Máx. & 1.00 & $.84-1.00$ & 1.00 & $.84-1.00$ & 1.00 & $.86-1.00$ & 1.00 & $.84-1.00$ \\
\hline & Prom & 1.00 & $.84-1.00$ & 1.00 & $.83-1.00$ & .99 & $.84-1.00$ & 1.00 & $.84-1.00$ \\
\hline \multirow[t]{3}{*}{ Metas } & Mín. & 1.00 & $.84-1.00$ & .97 & $.79-1.00$ & .93 & $.76-.98$ & 1.00 & $.84-1.00$ \\
\hline & Máx. & 1.00 & $.84-1.00$ & 1.00 & $.84-1.00$ & 1.00 & $.86-1.00$ & 1.00 & $.84-1.00$ \\
\hline & Prom & 1.00 & $.84-1.00$ & 1.00 & $.83-1.00$ & .96 & $.80-.99$ & 1.00 & $.84-1.00$ \\
\hline \multirow[t]{3}{*}{ Intereses } & Mín. & 1.00 & $.84-1.00$ & .97 & $.79-1.00$ & 1.00 & $.86-1.00$ & 1.00 & $.84-1.00$ \\
\hline & Máx. & 1.00 & $.84-1.00$ & 1.00 & $.84-1.00$ & 1.00 & $.86-1.00$ & 1.00 & $.84-1.00$ \\
\hline & Prom & 1.00 & $.84-1.00$ & 1.00 & $.83-1.00$ & 1.00 & $.86-1.00$ & 1.00 & $.84-1.00$ \\
\hline \multirow[t]{3}{*}{ Apoyo } & Mín. & 1.00 & $.84-1.00$ & 1.00 & $.84-1.00$ & 1.00 & $.86-1.00$ & 1.00 & $.84-1.00$ \\
\hline & Máx. & 1.00 & $.84-1.00$ & 1.00 & $.84-1.00$ & 1.00 & $.86-1.00$ & 1.00 & $.84-1.00$ \\
\hline & Prom & 1.00 & $.84-1.00$ & 1.00 & $.84-1.00$ & 1.00 & $.86-1.00$ & 1.00 & $.84-1.00$ \\
\hline \multirow[t]{3}{*}{ Barreras } & Mín. & 1.00 & $.84-1.00$ & .97 & $.79-1.00$ & .90 & $.72-.97$ & 1.00 & $.84-1.00$ \\
\hline & Máx. & 1.00 & $.84-1.00$ & 1.00 & $.84-1.00$ & 1.00 & $.86-1.00$ & 1.00 & $.84-1.00$ \\
\hline & Prom & 1.00 & $.84-1.00$ & .99 & $.82-1.00$ & .98 & $.83-1.00$ & 1.00 & $.84-1.00$ \\
\hline
\end{tabular}

Tabla 4. Resumen de la evaluación de la relevancia de los ítems, desde la percepción de los jueces

\begin{tabular}{|c|c|c|c|c|c|c|c|c|c|}
\hline \multirow{2}{*}{ Escalas } & & \multicolumn{2}{|c|}{ Argentina } & \multicolumn{2}{|c|}{ Colombia } & \multicolumn{2}{|c|}{ México } & \multicolumn{2}{|c|}{ Perú } \\
\hline & & $\mathrm{V}_{\text {Aiken }}$ & IC $95 \%$ & $\mathrm{~V}_{\text {Aiken }}$ & IC $95 \%$ & $\mathrm{~V}_{\text {Aiken }}$ & IC $95 \%$ & $\mathrm{~V}_{\text {Aiken }}$ & IC $95 \%$ \\
\hline \multirow{3}{*}{$\begin{array}{l}\text { Autoeficacia } \\
\text { percibida }\end{array}$} & Mín. & 1.00 & $.84-1.00$ & .97 & $.79-1.00$ & .83 & $.64-.93$ & .93 & $.74-.99$ \\
\hline & Máx. & 1.00 & $.84-1.00$ & 1.00 & $.84-1.00$ & 1.00 & $.86-1.00$ & 1.00 & $.84-1.00$ \\
\hline & Prom & 1.00 & $.84-1.00$ & 1.00 & $.83-1.00$ & .97 & $.82-.99$ & .99 & $.83-1.00$ \\
\hline \multirow{3}{*}{$\begin{array}{l}\text { Expectativa } \\
\text { de resultado }\end{array}$} & Mín. & 1.00 & $.84-1.00$ & 1.00 & $.84-1.00$ & .97 & $.81-.99$ & .97 & $.79-1.00$ \\
\hline & Máx. & 1.00 & $.84-1.00$ & 1.00 & $.84-1.00$ & 1.00 & $.86-1.00$ & 1.00 & $.84-1.00$ \\
\hline & Prom & 1.00 & $.84-1.00$ & 1.00 & $.84-1.00$ & .99 & $.84-1.00$ & 1.00 & $.83-1.00$ \\
\hline \multirow[t]{3}{*}{ Metas } & Mín. & 1.00 & $.84-1.00$ & .97 & $.79-1.00$ & .93 & $.76-.98$ & 1.00 & $.84-1.00$ \\
\hline & Máx. & 1.00 & $.84-1.00$ & 1.00 & $.84-1.00$ & 1.00 & $.86-1.00$ & 1.00 & $.84-1.00$ \\
\hline & Prom & 1.00 & $.84-1.00$ & 1.00 & $.83-1.00$ & .95 & $.78-.99$ & 1.00 & $.84-1.00$ \\
\hline \multirow[t]{3}{*}{ Intereses } & Mín. & 1.00 & $.84-1.00$ & .97 & $.79-1.00$ & .83 & $.64-.93$ & 1.00 & $.84-1.00$ \\
\hline & Máx. & 1.00 & $.84-1.00$ & 1.00 & $.84-1.00$ & 1.00 & $.86-1.00$ & 1.00 & $.84-1.00$ \\
\hline & Prom & 1.00 & $.84-1.00$ & 1.00 & $.83-1.00$ & .98 & $.83-.99$ & 1.00 & $.84-1.00$ \\
\hline \multirow[t]{3}{*}{ Apoyo } & Mín. & 1.00 & $.84-1.00$ & 1.00 & $.84-1.00$ & .87 & $.68-.95$ & 1.00 & $.84-1.00$ \\
\hline & Máx. & 1.00 & $.84-1.00$ & 1.00 & $.84-1.00$ & 1.00 & $.86-1.00$ & 1.00 & $.84-1.00$ \\
\hline & Prom & 1.00 & $.84-1.00$ & 1.00 & $.84-1.00$ & .99 & $.84-1.00$ & 1.00 & $.84-1.00$ \\
\hline \multirow[t]{3}{*}{ Barreras } & Mín. & 1.00 & $.84-1.00$ & .93 & $.74-.99$ & .93 & $.76-.98$ & 1.00 & $.84-1.00$ \\
\hline & Máx. & 1.00 & $.84-1.00$ & 1.00 & $.84-1.00$ & 1.00 & $.86-1.00$ & 1.00 & $.84-1.00$ \\
\hline & Prom & 1.00 & $.84-1.00$ & .98 & $.82-1.00$ & .99 & $.84-1.00$ & 1.00 & $.84-1.00$ \\
\hline
\end{tabular}

superioridad en Expectativas de resultado, Metas y Apoyo (Tabla 3). Respecto al criterio de relevancia percibida por los jueces se obtuvo que los valores de $\mathrm{V}$ de los ítems se ubican entre .83 [.64-.93] y 1[.84-1], con promedios más elevados en Argentina, Colombia y Perú. El promedio por escalas se encuentra entre .99 y 1 , con leve ventaja para Expectativas de resultado y Metas (Tabla 4).

\section{Discusión}

En respuesta a los escasos estudios en habla hispana sobre la autoeficacia vocacional (Casas \&
Blanco-Blanco, 2016), esta investigación inicial se dirigió al diseño y obtención de las evidencias de validez basada en el contenido, de una batería de autoeficacia vocacional para estudiantes de Psicología, que están por concluir su carrera profesional, que pueda utilizarse en los países de Argentina, Colombia, México y Perú. De ese modo se da respuesta a la necesidad de ampliar los estudios del modelo sociocognitivo de carrera de Lent a otras culturas (Lent \& Brown, 2017), maximizando su potencial generalización intercultural en una parte de Latinoamérica. 
La estructura interna de la batería diseñada, asume los supuestos teóricos del modelo sociocognitivo de desarrollo de carrera (Lent \& Brown, 2017; Lent, Brown, \& Hackett, 1994; 2002) y concuerda con las dimensiones que forman parte del constructo para probar el modelo teórico en el contexto de América anglosajona (Dutta et al., 2015; Lent et al., 2015; Lent, Lopez, Lopez, \& Sheu, 2008; Lent, Lopez, Sheu, \& Lopez, 2008; Lent, Sheu, Singley, Schmidt, Schmidt, \& Gloster, 2008; Morrow, Gore, \& Campbell, 1996; Sheu et al., 2010) y con otras regiones (Blanco, Casas, \& Mafokozi, 2016; Inda, Rodríguez, \& Peña, 2013). Sin embargo, no se asumió el modelo socio-cognitivo más reciente de autogestión profesional (Lent \& Brown, 2013; Lent et al., 2016), que si bien incluye las creencias de adaptabilidad profesional y de afrontamiento, excluye la dimensión de intereses, que amerita ser estudiada debido a que los universitarios, a lo largo de su formación profesional abandonan sus estudios y una de las causas fundamentales es la falta de vocación (Pérez, 2014; Lopez \& Beltrán, 2017; Zavala-Guirado, Álvarez, Vázquez, González, \& Bazán-Ramírez, 2018). Futuras investigaciones podrían comparar el modelo original con el de autogestión profesional para la población elegida; así como probar el carácter predictivo de la batería construida.

La valoración de los ítems, incluyó la posibilidad de que jueces (profesionales y estudiantes) brindaran observaciones $\mathrm{o}$ sugerencias, lo que permitió realizar modificaciones, eliminaciones y ajustes a los ítems evaluados; por lo que se reafirma que las metodologías mixtas proporcionan riqueza y sensibilidad al instrumento de recolección (BoschAlcaraz et al., 2018),

Se mantuvieron un total de 95 ítems con un límite inferior del intervalo de confianza menos restrictivo (Charter, 2003; Domínguez-Lara, 2017a; Merino-Soto, 2016) que permita obtener una mayor variabilidad, a fin de que sea posible, en un posterior estudio, seleccionar los mejores ítems mediante análisis factorial.

A su vez, se seleccionaron los ítems coherentes que poseían relevancia con las bases teóricas asumidas para el estudio (Moscoso \& Merino, 2017) y se descentró culturalmente el instrumento de medida (Tanzer, 2005), con la inclusión de términos comprensibles, coherentes y relevantes para los contextos investigados; sin embargo, a pesar de ello es necesario obtener nuevas evidencias de validez basadas en el contenido en otros contextos (Muñiz \& FonsecaPedrero, 2008; Sánchez-Mendiola, 2016; Zumbo, 2007) como el Latinoamericano, considerando que después de EEUU las investigaciones sobre el modelo se han llevado a cabo en países asiáticos y europeos (Sheu \& Bordon, 2017).

Los criterios con los valores más elevados en el límite inferior fueron los de coherencia $\mathrm{y}$ relevancia, que han sido valorados por expertos, cumpliendo con las exigencias de la elaboración de instrumentos (Muñiz \& Fonseca-Pedrero, 2008). En cambio, la claridad de los ítems fue valorada por los estudiantes, ya que se considera fundamental que los reactivos sean comprensibles para la población a la que se dirigirá el instrumento (Hareendran et al, 2017; Merino-Soto, 2016; Merino-Soto \& Fernández-Arata, 2017).

El estudio presentado aquí empleó la metodología cuantitativa para obtener y reportar la validez de contenido. Debido a limitaciones idiosincrásicas de la presente investigación (e.g., disponibilidad completa de los jueces, un marco restringido de tiempo y enfoque de los investigadores), los resultados de esta fase de la investigación han maximizado aspectos que deben ser complementados con estrategias cualitativas; esto puede ser muy conveniente si se integran ambos enfoques en un diseño de metodología mixta.

Los resultados del presente estudio deben interpretarse en el contexto de sus limitaciones. No se homologó la cantidad de los estudiantes por sexo, que participaron como jueces para valorar la claridad de los ítems, considerando que la mayoría fueron mujeres. Sin embargo, la mayor proporción de mujeres parece ser habitual en la carrera de psicología en el contexto internacional, y particularmente, en los países participantes. Tampoco fue efectuada una exploración cualitativa que sirva para triangular los resultados obtenidos del enfoque cuantitativo; los estudios posteriores podrían complementar con mayor profundidad los resultados mediante técnicas cualitativas, como el método Delphi (Gil-Gómez \& Pascual-Ezama, 2012), grupos focales o entrevistas cognitivas. 
La investigación se circunscribe exclusivamente a estudiantes de psicología, y el contenido muestreado es relevante a las competencias esperadas e integradas en su perfil profesional (González, González, \& Vicencio, 2014; Lunt, Peiró, Poortinga, \& Roe, 2015; Red Psicología Proyecto MECESUP ULS 0601, 2013); por tanto, futuros estudios que deseen efectuarse en otros campos profesionales, deberán reformular los contenidos vinculados especialmente a la autoeficacia percibida y realizar la adaptación psicométrica respectiva.

Además, considerando que otras evidencias de validez no fueron obtenidas, y esto es condicional para asegurar la representación del constructo, así como su capacidad descriptiva, será necesario encauzar nuevos estudios que permitan obtener las evidencias de validez basada en la estructura interna (American Educational Research Association, American Psychological Association \& National Council on Measurement in Education, 1985); la comprobación, en contextos distintos, de las evidencias métricas (Elosua, 2003; Merino-Soto \& Calderón-De la Cruz, 2018) e invarianza factorial (Barrera-Barrera, NavarroGarcía, \& Peris-Ortiz, 2015; Gálvez-Nieto, Salvo, Trizano-Hermosila, Hederich, \& Polanco, 2018; Leal-Soto \& Alonso-Tapia, 2017), así como también, obtener evidencias externas de validez (Domínguez-Lara, 2018); y, hallar la capacidad predictiva de la batería de instrumentos sobre el desempeño pre-profesional.

Se concluye que la batería sociocognitiva de autoeficacia vocacional, construida para estudiantes de Psicología que concluyen la carrera, presenta su primera evidencia de validez basada en el contenido en el contexto argentino, colombiano, mexicano y peruano. Finalmente, la construcción intercultural de la medida posibilita atender la necesidad de integrar la teoría, la investigación y la práctica (Lent, 2017) y seguir contribuyendo a la Psicología de desarrollo de carrera.

\section{Referencias}

Abad, F., García, C., Gil, B., Olea, J., Ponsoda, V., \& Revuelta, J. (2004). Introducción a la Psicometría. Teoría clásica de los tests y teoría de la respuesta al ítem Madrid: Universidad Autónoma de Madrid. Recuperado de https://www.uam.es/personal_pdi/psicologia/c adalso/Docencia/Psicometria/Apuntes/tema1T yP_4.pdf

Aiken, L. R. (1980). Content validity and reliability of single items or questionnaires. Educational and Psychological Measurement, 40, 955-959. doi:10.1177/001316448004000419

Aiken, L. R. (1985). Three coefficients for analyzing the reliability and validity of ratings. Educational and Psychological Measurement, $\quad 45, \quad$ 131-142. doi: $10.1177 / 0013164485451012$

Ali, S., \& Brown, S. (2017). Integration of theory, research, and practice: Using our tools to address challenging times. In J. P. Sampson, E. Bullock-Yowell, V. C. Dozier, D. S. Osborn, \& J. G. Lenz (Eds.), Integrating theory, research, and practice in vocational psychology: Current status and future directions (73-76). Tallahassee, FL: Florida State University. doi:10.17125/svp2016.ch6

American Educational Research Association, American Psychological Association \& National Council on Measurement in Education. (1985). Standards for educational and psychological testing. Standards for educational and psychological testing. Washington, D.C.: American Psychological Association.

Ato, M., López, J., \& Benavente, A. (2013). Un sistema de clasificación de los diseños de investigación en psicología. Anales de Psicología, 29(3), 1038-1059. doi:10.6018/analesps.29.3.178511

Aydin, A., \& Pasinlioglu, T. (2018). Reliability and validity of a Turkish version of the Prenatal Breastfeeding Self-Efficacy Scale. Midwifery, $\quad 64, \quad$ 11-16. doi:10.1016/j.midw.2018.05.007

Bandura, A. (1977). Self-efficacy: Toward a unifying theory of behavioral change. Psychological Review, 84(1), 191-215. doi:10.1037/0033-295X.84.2.191

Bandura, A. (1982). Self-efficacy mechanism in human agency. American Psychologist, 37, 122147. doi:10.1037/0003-066X.37.2.122 
Bandura, A. (1986). Social foundations of thought and action. Englewood Cliff, N.J: Prentice Hall.

Bandura, A. (1997). Self-efficacy. The exercise of control. New York: Freeman and Company.

Bandura, A. (1998). Health promotion from the perspective of social cognitive theory. Psychology \& Health, 13(4), 623-649. doi:10.1080/08870449808407422

Bandura, A. (2008). A evolução da teoria social cognitiva. Em A. Bandura, R. Azzi, \&, R. G. Polydoro, S. Teoria Social Cognitiva: conceitos básicos (15-41). Porto Alegre: ArtMed.

Bandura, A. (2010). Self-efficacy. The Corsini encyclopedia of psychology. doi:10.1002/9780470479216.corpsy083

Barrera-Barrera, R., Navarro-García, A., \& PerisOrtiz, M. (2015). El papel de la invarianza factorial en la validación del constructo calidad de servicio electrónico. Revista Europea de Dirección y Economía de la Empresa, 24, 1324. doi:10.1016/j.redee.2014.07.001

Beauchamp, M. R., Crawford, K. L., \& Jackson, B. (2018). Social cognitive theory and physical activity: Mechanisms of behavior change, critique, and legacy. Psychology of Sport and Exercise. doi:10.1016/j.psychsport.2018.11.009

Betz, N. E., \& Taylor, K. M. (2012). Career Decision Self-Efficacy Scale manual. Redwood City, CA: Mindgarden Inc.

Blanco, A., Casas, Y., \& Mafokozi, J. (2016). Adaptación y propiedades psicométricas de escalas sociocognitivas. Una aplicación en el ámbito vocacional científico-matemático. Revista Española de Orientación y Psicopedagogía, 27(1), 8-28. doi:10.5944/reop.vol.27.num.1.2016.17005

Blustein, D. (2017). Integrating theory, research, and practice: Lessons learned from the evolution of vocational psychology. In J. P. Sampson, E. Bullock-Yowell, V. C. Dozier, D. S. Osborn, \& J. G. Lenz (Eds.), Integrating theory, research, and practice in vocational psychology: Current status and future directions (179-187). Tallahassee, FL: Florida State University. doi:10.17125/svp2016.ch19

Bosch-Alcaraz, A., Jordan-Garcia, I., AlcoleaMonge, S., Fernández-Lorenzo, R.,
Carrasquer-Feixa, E., Ferrer-Orona, M., \& Falcó-Pegueroles, A. (2018). Validez de contenido de una escala de confort crítico pediátrico mediante una metodología mixta. Enfermería Intensiva, 29(1), 21-31. doi:10.1016/j.enfi.2017.04.003

Brusokas, A., \& Malinauskas, R. (2014). Career Self-efficacy among Lithuanian Adolescents in Sports Schools. Procedia - Social and Behavioral Sciences, 116(21), 212-16. doi:10.1016/j.sbspro.2014.01.196

Carbonero, M., \& Merino Tejedor, E. (2002). La Escala de Autoeficacia Vocacional: Desarrollo, análisis y aplicaciones del instrumento. Revista de Psicodidáctica, 14, 99-114.

Carretero-Dios, H., \& Pérez, C. (2007). Normas para el desarrollo y revisión de estudios instrumentales: Consideraciones sobre la selección de tests en la investigación psicológica. International Journal of Clinical and Health Psychology, 7(3), 863-882.

Casas, Y., \& Blanco-Blanco, A. (2016). Una revisión de la investigación educativa sobre autoeficacia y teoría cognitivo social en Hispanoamérica. Bordón, Revista de Pedagogía, 68(4), 27-47.

Charter, R. A. (2003). A breakdown of reliability coefficients by test type and reliability method, and the clinical implications of low reliability. The Journal of General Psychology, 130(3), 290-304. doi:10.1080/00221300309601160

Chen-Tsang, T., Hsuan, H., \& Chun-Chin, Y. (2017). Career decision self-efficacy plays a crucial role in hospitality undergraduates' internship efficacy and career preparation. Journal of Hospitality, Leisure, Sport \& Tourism Education, 21, 61-68. doi:10.1016/j.jhlste.2017.08.002

Cicchetti, D. V. (1994). Guidelines, criteria, and rules of thumb for evaluating normed and standardized assessment instruments in psychology. Psychological Assessment, 6, 284-290. doi:10.1037/1040-3590.6.4.284

Domínguez-Lara, S. (2017a). Construcción de una escala de autoeficacia para la investigación: Primeras evidencias de validez. Revista Digital de Investigación en Docencia Universitaria, RIDU, 11(2), 308 -332. 
Recuperado

de

http://www.scielo.org.pe/scielo.php?script=sci _arttext\&pid=S2223-

$25162017000200019 \& \operatorname{lng}=$ es\&tlng=es.

Domínguez-Lara, S. (2017b). Autorreporte del rendimiento en exámenes: Un informe exploratorio. Investigación en Educación Médica, 6(23), 215-217. doi:10.1016/j.riem.2016.09.003

Domínguez-Lara, S. (2018). Ítem único de ansiedad ante exámenes: Evidencias de validez convergente e incremental en estudiantes universitarios. Educación Médica, 19(5),

264-270. doi:10.1016/j.edumed.2017.04.004

Dutta, A., Kang, H., Kaya, C., Flowers, S. Sharp, S., Chan, F., \& Da Silva, E. (2015). SocialCognitive Career Theory predictors of STEM career interests and goal persistence in minority college students with disabilities: A path analysis. Journal of Vocational Rehabilitation, 43, 159-167. doi:10.3233/JVR-150765

Elosua, P. (2003). Sobre la validez de los tests. Psicothema, 15(2), 315-321.

Escurra, L. M. (1988). Cuantificación de la validez de contenido por criterio de jueces. Revista de Psicología, 6(1-2), 103-111.

Gálvez-Nieto, J. L., Salvo, S., Trizano-Hermosila, I., Hederich, C., \& Polanco, K. (2018). Equivalencia factorial de la Escala de Actitudes hacia la Autoridad Institucional en Adolescentes (AAI-A) chilenos y colombianos. Revista Iberoamericana de Diagnóstico y Evaluación - e Avaliação Psicológica, $\quad$ 1(46), $109-119$. doi:10.21865/RIDEP46.1.08

García-Jiménez, E. (2016). Concepto de excelencia en enseñanza superior universitaria. Educación Médica, 17(3), 8387. doi:10.1016/j.edumed.2016.06.003

García, P., Restubog, S. Bordia, S., \& Roxas, R. (2015). Career optimism: The roles of contextual support and career decisionmaking self-efficacy. Journal of Vocational Behavior, 88 ,

10-18. doi:10.1016/j.jvb.2015.02.004

Gil-Gómez, B., \& Pascual-Ezama, D. (2012). La metodología Delphi como técnica de estudio de la validez de contenido. Anales de
Psicología, 28(3), 1011-1020. Recuperado de http://www.redalyc.org/pdf/167/16723774041 .pdf

González, M., González, I., \& Vicencio, K. (2014). Descripción del rol autopercibido del psicólogo y sus implicancias en los procesos de formación de pregrado. Psicoperspectivas, 13(1), 108-120. doi:10.5027/psicoperspectivas-Vol13-Issue1fulltext-296

Hareendran, A., Mannix, S., Skalicky, A., Bayliss, M., Blumenfeld, A., Buse, D., Desai, P, Ortmeier, B., \& Sapra, S. (2017). Development and exploration of the content validity of a patient-reportes outcome measure to evaluate the impact of migraine-the migraine physical function impact diary (MPFID). Health and Quality of Life Outcomes, 15, 224. doi:10.1186/s12955-017-0799-1

Howard, K. (2017). The role of refereed journals in integrating theory, research and practice directions. In J. P. Sampson, E. BullockYowell, V. C. Dozier, D. S. Osborn, \& J. G. Lenz (Eds.). Integrating Theory, Research, and Practice in Vocational Psychology: Current Status and future directions (175178). Tallahassee, FL: Florida State University.

Inda, M., Rodríguez, C., \& Peña, J. (2013). Gender differences in applying social cognitive career theory in engineering students. Journal of Vocational Behavior, 83, 346-355. doi:10.1016/j.jvb.2013.06.010

Kane, M. T. (2013). Validating the interpretations and uses of test scores. Journal of Educational Measurement, $\quad 50, \quad 1-73$. doi:10.1111/jedm. 12000

Leal-Soto, F., \& Alonso-Tapia, J. (2017). Cuestionario de Clima Motivacional de la Clase: Validez intercultural, intergénero, evolutiva y predictiva. Revista Iberoamericana de Diagnóstico y Evaluación - e Avaliação Psicológica, 3(45), 57-70. doi:10.21865/RIDEP45.3.05

Lent, R. (2005). A social cognitive view of career development and counseling. En S. D. Brown y R. T. Lent (Eds.), Career development and counseling: Putting theory and research to work (pp. 101-127). Hoboken, NJ: Wiley. 
Lent, R. (2017). Integration of theory, research, and practice: A social cognitive perspective. In J. P. Sampson, E. Bullock-Yowell, V. C. Dozier, D. S. Osborn, \& J. G. Lenz (Eds.). Integrating theory, research, and practice in vocational psychology: Current Status and future directions (20-26). Tallahassee, FL: Florida State University. doi:10.17125/svp2016.ch17

Lent, R. W., \& Brown, S. D. (2006). Integrating person and situation perspectives on work satisfaction: A social-cognitive view. Journal of Vocational Behavior, 69(2), 236-247. doi:10.1016/j.jvb.2006.02.006

Lent, R. W., \& Brown, S. D. (2013). Social cognitive model of career self-management: Toward a unifying view of adaptive career behavior across the life span. Journal of Counseling Psychology, 60, 557-568. doi: $10.1037 / \mathrm{a} 0033446$

Lent, R., \& Brown, S. D. (2017). Social cognitive career theory in a diverse world: guest editor's introduction. Journal of Career Assessment, 25(1), 3-5. doi:10.1177/1069072716657811

Lent, R., Brown, D., \& Hackett, G. (1994). Toward a unifying social cognitive theory of career and academic interest, choice, and performance. Journal of Vocational Behavior, 45, 79-122. doi:10.1006/jvbe.1994.1027

Lent, R., Brown, S., \& Hackett, G. (2002). Social cognitive theory (255-311). In D. Brown \& associates. Career choice and development. (4 ${ }^{\text {th }}$ ed.). New York: Wiley.

Lent, R. W., Brown, S. D., \& Larkin, K. C. (1986). Self-efficacy in the prediction of academic performance and perceived career options. Journal of Counseling Psychology, 33(3), 265-269. doi:10.1037/0022-0167.33.3.265

Lent, R., Brown, S. D., Schmidt, J., Brenner, B., Lyons, H., \& Treistman, D. (2003). Relation of contextual supports and barriers to choice behavior in engineering majors: Test of alternative social cognitive models. Journal of Counseling Psychology, 50(4), 458-465. doi:10.1037/0022-0167.50.4.458

Lent, R., Ezeofor, I., Morrison, A., Penn, L., \& Ireland, G. (2016). Applying the social cognitive model of career self-management to career exploration and decision-making.
Journal of Vocational Behavior, 93, 47-57. doi:10.1016/j.jvb.2015.12.007

Lent, R., \& Hackett, G. (1987). Career selfefficacy: Empirical status and future directions. Journal of Vocational Behavior 30, 347-382. doi:10.1016/0001-8791(87)90010-8

Lent, R., Hackett, G., \& Brown, S. (2004). Una perspectiva social cognitiva de la transición entre la escuela y el trabajo. Evaluar, 4, 1-22.

Lent, R., Lopez, A., Lopez, F., \& Sheu, H. (2008). Social cognitive career theory and the prediction of interests and choice goals in the computing disciplines. Journal of Vocational Behavior, 73(1), 52-62. doi:10.1016/j.jvb.2008.01.002

Lent, R., Lopez, F., Sheu, H., \& Lopez, A. (2008). Social cognitive predictors of the interests and choices of computing majors: Applicability to underrepresented students. Journal of Vocational Behavior, 78, 184-192. doi:10.1016/j.jvb.2010.10.006

Lent, R., Miller, M., Smith, P., Watford, B., Hui, K., \& Lim, R. (2015). Social cognitive model of adjustment to engineering majors: Longitudinal test across gender and race/ethnicity. Journal of Vocational $\begin{array}{lll}\text { Behavior, } & \text { 86, } & \text { 77-85. }\end{array}$ doi:10.1016/j.jvb.2014.11.004

Lent, R., Sheu, H., Singley, D., Schmidt, J., Schmidt, L., \& Gloster, C. (2008). Longitudinal relations of self-efficacy to outcome expectations, interests, and major choice goals in engineering students. Journal of Vocational Behavior, 73, 328-335. doi:10.1016/j.jvb.2008.07.005

Leung, A. (2008). The big five career theories. En J. A. Athanasou \& R. Van Esbroeck (eds.) International Handbook of Career Guidance (115-132). Hong Kong, China: The Chinese University of Hong Kong.

Lopez, L., \& Beltrán, A. (2017). La deserción en estudiantes de educación superior: Tres percepciones en estudio, alumnos, docentes y padres de familia. Pistas Educativas, 126, 143-159.

Lunt, I., Peiró, J. M., Poortinga, Y., \& Roe, R. (2015). EuroPsy: Standards and quality in education for professional psychologists. Boston: Hogrefe. 
Makransky, G., Rogers, M., \& Creed, P. (2014). Analysis of the construct validity and measurement invariance of the Career Decision Self-Efficacy Scale. A Rasch model approach. Journal of Career Assessment, 23(4), 645-660. doi: $10.1177 / 1069072714553555$

Moscoso, M., \& Merino, C. (2017). Construcción y validez de contenido del Inventario de Mindfulness y Ecuanimidad: Una perspectiva iberoamericana. Mindfulness \& Compassion, 2(1), 9-16. doi:10.1016/j.mincom.2017.01.001

Merino-Soto, C. (2016). Percepción de la claridad de los ítems: Comparación del juicio de estudiantes y jueces-expertos. Revista Latinoamericana de Ciencias Sociales, Niñez $y \quad$ Juventud, 14(2), 1469-1477. doi:10.11600/1692715x.14239120615

Merino-Soto, C., \& Calderón-De la Cruz, G. (2018). Validez de estudios peruanos sobre el estrés y burnout. Revista Peruana de Medicina Experimental y Salud Pública, 35(2), $1-2$. doi:10.17843/rpmesp.2018.352.3521.

Merino-Soto, C., \& Fernández-Arata, M. (2017). Ítem único de burnout en estudiantes de educación superior: Estudio de validez de contenido. Educación Médica, 18(3), 195198. doi:10.1016/j.edumed.2016.06.019

Merino, C., \& Livia, J. (2009). Intervalos de confianza asimétricos para el índice de la validez de contenido: Un programa visual basic para la $\mathrm{V}$ de Aiken. Anales de Psicología, 25(1), 169-171. Recuperado de https://revistas.um.es/analesps/article/view/71 631

Miguel, J., Silva, J., \& Prieto, G. (2013). Career Decision Self-Efficacy Scale - Short Form: A Rasch analysis of the Portuguese version. Journal of Vocational Behavior, 82(2), 116123. doi:10.1016/j.jvb.2012.12.001

Morrow, S., Gore, P., \& Campbell, B. (1996). The application of a sociocognitive framework to the career development of lesbian women and gay men. Journal of Vocational Behaviour, 48, 136-148. doi:10.1006/jvbe.1996.0014

Muñiz, J., \& Fonseca-Pedrero, E. (2008). Construcción de instrumentos de medida para la evaluación universitaria. Revista de Investigación en Educación, 5, 13-25.
Penfield, R. D., \& Giacobbi, P. R. (2004). Applying a score confidence interval to Aiken's item content-relevance index. Measurement in Physical Education and Exercise Science, 8(4), 213-225. doi:10.1207/s15327841mpee0804_3

Pérez, C. (2014). Deserción universitaria, analizada desde la experiencia del Departamento de atención psicopedagógica. IV Conferencia Latinoamericana sobre el abandono en la educación superior. Universidad Don Bosco, El Salvador.

Porter, L. S., Keefe, F. J., Wellington, C., \& de Williams, A. (2008). Pain communication in the context of osteoarthritis: Patient and partner self-efficacy for pain communication and holding back from discussion of pain and arthritis-related concerns. The Clinical Journal of Pain, 24(8), 662-668. doi:10.1097/ajp.0b013e31816ed964

Red Psicología Proyecto MECESUP ULS 0601 (2013). Competencias del perfil de egreso de psicólogo. En M. T. Juliá (Ed.), Competencias del psicólogo en Chile. Propuestas desde las universidades estatales (pp. 166-188). La Serena: Universidad de La Serena.

Sánchez-Mendiola, M. (2016). Mi instrumento es más válido que el tuyo: ¿Por qué seguimos usando ideas obsoletas? Investigación en Educación Médica, 5(19), 133-135. doi:10.1016/j.riem.2016.05.007

Sheu, H. B., \& Bordon, J. J. (2017). SCCT Research in the international context: Empirical evidence, future directions, and practical implications. Journal of Career Assessment, $\quad 25(1), \quad 58-74$. doi:10.1177/1069072716657826

Sheu, H., Lent, R., Brown, S. D., Miller, M. Hennessy, K., \& Duffy, R. (2010). Testing the choice model of social cognitive career theory across Holland themes: A meta-analytic path analysis. Journal of Vocational Behavior, 76, 252-264. doi:10.1016/j.jvb.2009.10.015

Tanzer, N. K. (2005). Developing tests for use in multiple languages and cultures: A plea for simultaneous development. In Hambleton, R. K., Merenda, P. F., Spielberger, C. D. (Eds.), Adapting educational and psychological tests for cross-cultural assessment (235-263). Mahwah, NJ: Erlbaum. 
Tschannen-Moran, M., \& Hoy, A. W. (2001). Teacher efficacy: capturing an elusive construct. Teaching and Teacher Education, 17(7), 783-805.

doi:10.1016/s0742-051x(01)00036-1

Urbanaviciute, I., Pociute, B. Kairys, A., \& Liniauskaite, A. (2016). Perceived career barriers and vocational outcomes among university undergraduates: Exploring mediation and moderation effects. Journal of Vocational Behavior, 92, 12-21. doi:10.1016/j.jvb.2015.11.001

Volodina, A., \& Nagy, G. (2016). Vocational choices in adolescence: The role of gender, school achievement, self-concepts, and vocational interests. Journal of Vocational Behavior, 95-96, 58-73. doi:10.1016/j.jvb.2016.07.005

Wolf, B., Herrmann, M., \& Brandstätter, V. (2018). Self-efficacy vs. action orientation: Comparing and contrasting two determinants of goal setting and goal striving. Journal of Research in Personality, 73, 35-45. doi:10.1016/j.jrp.2017.11.001

Wood, R., \& Bandura, R. (1989). Social cognitive theory of Organizational Management. Academy of Management Review, 14(3), 361384. doi:10.5465/amr.1989.4279067

Zavala-Guirado, M., Álvarez, M., Vázquez, M., González, I., \& Bazán-Ramírez, A. (2018). Factores internos, externos y bilaterales asociados con la deserción en estudiantes universitarios. Interacciones, 4(1), 59-69. doi:10.24016/2018.v4n1.103

Zimmerman, B. J., Bandura, A., \& Martinez-Pons, M. (1992). Self-motivation for academic attainment: The role of self-efficacy beliefs and personal goal setting. American Educational Research Journal, 29(3), 663676. doi:10.3102/00028312029003663

Zumbo, B. D. (2007). Validity: Foundational issues and statistical methodology. In C. R. Rao \& S. Sinharay (Eds.), Handbook of statistics: Vol. 26. Psychometrics (pp. 45-79). Amsterdam, Netherlands: Elsevier Science. doi:10.1016/S0169-7161(06)26003-6 